\title{
Global properties of the population of HII regions in NGC 7479 from photometric $\mathrm{H} \alpha$ imaging
}

\author{
M. Rozas ${ }^{1}$, A. Zurita ${ }^{1}$, C.H. Heller ${ }^{2}$, and J.E. Beckman ${ }^{1}$ \\ 1 Instituto de Astrofísica de Canarias, E-38200 La Laguna, Tenerife, Canarias, Spain \\ 2 Universitäts Sternwarte, Geismarlandstra $\beta$ e, 11, D-37083 Göttingen, Germany
}

Received August 14; accepted October 9, 1998

\begin{abstract}
We present a new high quality continuumsubtracted $\mathrm{H} \alpha$ image of NGC 7479. Using a novel semiautomated technique we have determined the positions, angular sizes, and absolute fluxes of over 1000 HII regions and have constructed the luminosity function for the regions over the whole galaxy, showing that its slope is within the published range for spirals of the same morphological type. However NGC 7479 is notable in that its bar shows unusually strong star formation along the whole structure. This prompted us to undertake separate analyses of the Hil regions in the bar and in the disc. The disc luminosity function (LF) shows clean bi-linear behaviour as found previously in late-type spirals, with a break at $\log L_{\mathrm{H} \alpha}=38.6 \mathrm{erg} \mathrm{s}^{-1}$, whereas the bar LF shows a much less regular form. The difference is not due to the small numbers of Hil regions in the bar, but reflects a physical difference between the bar and the disc in the properties of their sets of regions. We show separate plots of the parameters for HiI regions in the bar and the disc selected for their regular shapes and absence of blending.

We have derived galaxy-wide relations for the HiI region set: the diameter distribution function, luminosity versus volume and number versus luminosity versus diameter, and deduced the global $\mathrm{H} \alpha$ surface density distribution and disc scale length. Measuring the integrated diffuse $\mathrm{H} \alpha$ flux for the galaxy, we compare this with the computed ionizing flux escaping from the population of density-bounded HII regions, finding that both in terms of energy balance and of geometry, the hypothesis that this escaping flux gives rise to the diffuse $\mathrm{H} \alpha$ is well borne out in NGC 7479, and that a significant fraction of this flux escapes completely into the intergalactic medium.
\end{abstract}

Key words: galaxies: individual (NGC 7479) — galaxies: spiral — galaxies: ISM

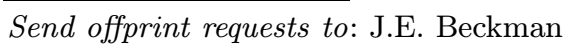

\section{Introduction}

Since the work of Hodge and Kennicutt in the 1970's and 1980's (e.g. Hodge 1976, 1987; Kennicutt 1984, 1989; Kennicutt et al. 1989) it has become clear that the set of HiI regions in a spiral, or irregular galaxy, is worth studying as a population, i.e. for its statistical properties as well as for the physical properties of the individual regions. As well as being of key importance for the energy balance of the interstellar medium as a whole, the HiI region population reflects, in a manner readily observable, the parameters of the massive exciting stars within the regions, and is therefore of interest in the continuing study of the star formation rate (SFR) and the initial mass function (IMF) at the massive end of the stellar mass range (Kennicutt 1992). The number of complete studies of this type since the beginning of the CCD era is restricted. We ourselves have contributed a series of studies of the statistical properties of HII regions in NGC 4321 (Cepa \& Beckman 1990), NGC 3992 (Cepa \& Beckman 1989), NGC 6814 (Knapen et al. 1993), and of NGC 157, NGC 3631, NGC 6764 and NGC 6951 (Rozas et al. 1996; Rozas et al. 1996). In these papers we concentrated mainly on the HiI region luminosity functions (LF's) in $\mathrm{H} \alpha$ and their global properties, as well as the disc-wide geometrical distribution of emission, although in Rozas et al. (1996b), Beckman et al. (1999), as well as in Rozas et al. (1998) we derived the internal physical parameters: emission measure, electron density, and total mass of representative samples of, especially, the most luminous regions. Other studies based on statistical properties of HiI regions in spiral galaxies are found in Rand (1992) and Knapen (1998).

Here we offer the most complete study to date of the HII regions in a single galaxy: NGC 7479, in which all the techniques developed in the previous series of papers have been brought to bear. NGC 7479 is a barred spiral, of type SBc (from de Vaucouleurs et al. 1991; hereafter RC3), with a particularly well defined and well developed bar, which virtually dominates the luminosity of the galaxy, 
emitting almost $50 \%$ of its energy in any representative optical band (Blackman 1983). Its arm structure is asymmetric and this asymmetry has been reasonably ascribed to the after-effects of a recent merger with a low mass companion (Mihos \& Hernquist 1994; Laine 1997; Laine \& Heller, in preparation). We list its basic parameters in Table 1, from RC3, except for the position angle and the inclination of the galaxy, taken from Laine \& Gottesman (1998). This study is not designed to differentiate between galaxies which have recently suffered a merger and those which have not. The global properties of the HiI regions in NGC 7479 do in fact show some interesting differences from the series of galaxies cited in the preceding paragraph, and we show that this is due to the major star formation zones along the bar, which may have been affected by the merger. We will itemize the differences and discuss them in detail in the text. However many of the basic parameters of the population of HiI regions do not differ greatly from those in other galaxies. In particular the LF, at luminosities above the completeness limit, approximates a power law with exponent not far from 2, as found by Kennicutt et al. (1989) for a set of 30 galaxies of different morphological types, using pre-CCD data. However, the studies by Rozas et al. (1996a,b), Knapen et al. (1993), cited above, based on CCD data of excellent $\mathrm{S} / \mathrm{N}$ and angular resolution, revealed a local peak at $\log L_{\mathrm{Str}}=38.6\left(\mathrm{erg} \mathrm{s}^{-1}\right)$ (which we have termed the Strömgren luminosity) in the LF of each of the galaxies treated, accompanied by a change in slope, which we have attributed to the transition from ionization bounding at lower luminosities to density bounding at higher luminosities (Rozas et al. 1998; Beckman et al. 1999). An initial objective of the present study was to see if such a "glitch" exists in the LF of NGC 7479. Further objectives were to measure the basic physical properties of the HiI regions, to see whether the properties of those along the bar differ measurably from these in the disc.

Table 1. NGC 7479: Basic parameters

\begin{tabular}{ll}
\hline R.A. $(2000)$ & $23^{\mathrm{h}} 04^{\mathrm{m}} 57^{\mathrm{S}} .1$ \\
Dec $(2000)$ & $12^{\circ} 19^{\prime} 18^{\prime \prime}$ \\
Type & SBS 5 \\
$D(\mathrm{Mpc})$ & 31.94 \\
$r_{25}$ & $2.04^{\prime}$ \\
$B_{\mathrm{T}}$ & 11.6 \\
$v_{\text {opt }}$ & $2394 \mathrm{~km} \mathrm{~s}^{-1}$ \\
$i$ & $51^{\circ}$ \\
PA & $22^{\circ}$ \\
\hline
\end{tabular}

In Sect. 2 of the paper we describe the observations and their reduction, and the preparation of the catalogue of HII regions: $\mathrm{H} \alpha$ fluxes, positions, and diameters. This section is rather less routine than is customary, since in all our previous work we used interactive region by region integration to obtain the fluxes of the many hundreds of HiI regions in a given galaxy, but here we have used for the first time a semi-automatic method, which saves a major fraction of the time, and will be needed for multigalaxy statistical studies. In Sect. 3 we derive the LF's. In Sects. 4 and 5 we produce diameter and flux distributions, and in Sect. 6 we show the luminosity volume relations. The derivation of the physical parameters of individual regions is described in Sect. 7, with their resulting values. Number-luminosity-diameter relation is described in Sect. 8. In Sect. 9 we compare the measured diffuse $\mathrm{H} \alpha$ flux in the disc of NGC 7479 with the computed ionizing flux escaping from the density bounded HII regions, testing, both in terms of energy balance and geometry, the hypothesis that this escaping flux produces the diffuse $\mathrm{H} \alpha$; and in Sect. 10 we draw our statistical and physical conclusions.

\section{Observations, data reduction and the production of the HII region catalogue}

The observations of NGC 7479 were made during the night of May 20 th 1992 with the $4.2 \mathrm{~m}$ William Herschel Telescope (WHT) on La Palma. We used the TAURUS instrument in imaging mode as a re-imaging camera, mounted at the Cassegrain focus of the telescope. The detector used was an EEV CCD 7 with projected pixel size $0^{\prime \prime} .279 \times 0$ ' .279 . Observing conditions were very good with 0 ". 8 seeing (FWHM as measured in the final images) and photometric sky. We obtained two exposures of 1200 seconds: one through a $15 \AA\left(\sim 685 \mathrm{~km} \mathrm{~s}^{-1}\right)$ wide filter whose central wavelength coincided with that of the redshifted $\mathrm{H} \alpha$ emission from the galaxy, and another through a nonredshifted $\mathrm{H} \alpha$ filter $(\lambda 6565 \AA$ with $15 \AA$ width) for continuum subtraction.

Standard reduction routines were used; bias level was first subtracted, and the images were then corrected using appropriate dawn sky flatfields. Next, the images were aligned and cleaned of cosmic ray effects and then the continuum image was subtracted from the line $(\mathrm{H} \alpha+$ continuum) image, giving the net $\mathrm{H} \alpha$ flux. The procedure is described with more detail in Rozas et al. (1996a).

Absolute flux calibration was carried out using observations of standard stars from the lists of Oke (1974), Stone (1977), and Filippenko \& Greenstein (1984). The luminosity in $\mathrm{H} \alpha$, corresponding to a single instrumental count is $9.2710^{34} \mathrm{erg} \mathrm{s}^{-1}$ count $^{-1}$.

Before producing the HII region catalogues, we flagged the foreground stars in the image. These are distinguishable from HiI regions by their regular, circular shapes in the original, unsubtracted image, and because they show much more intensity in the continuum than in the corresponding $\mathrm{H} \alpha$ continuum-subtracted image. (Ideally, foreground stars should not show up at all in the $\mathrm{H} \alpha$ continuum-subtracted image, but in most cases some residual is seen, due to e.g. differences in point spread 


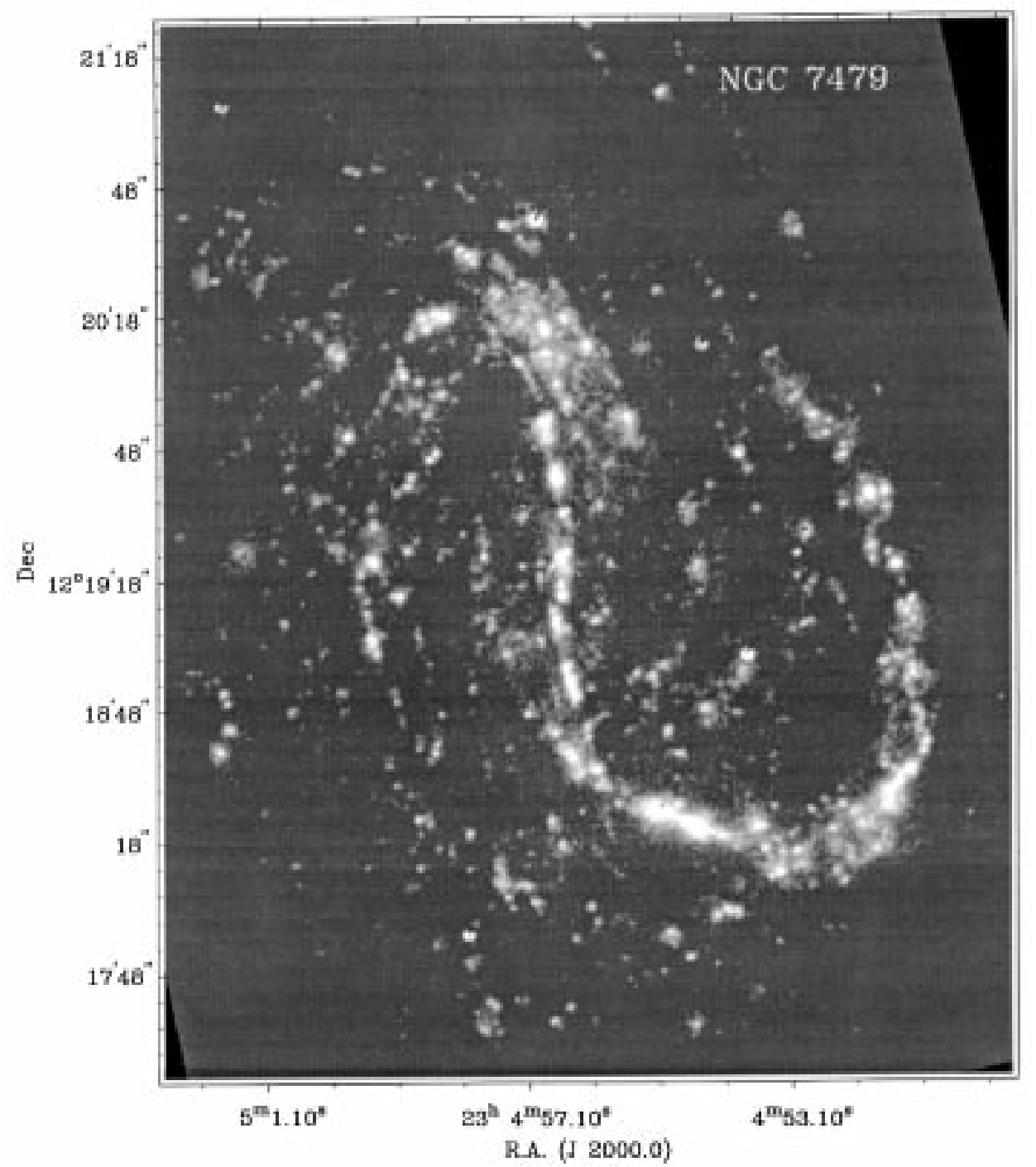

Fig. 1. Grey scale representation of the $\mathrm{H} \alpha$ continuum-subtracted image of NGC 7479

function, alignment, or stellar emission between the line and continuum image; or because the star is saturated in one or both images.) Emission in the $\mathrm{H} \alpha$ image coincident with a foreground star on the continuum image was considered residual starlight, and not entered in the catalogue as an HiI region. Any HII region with a superposed foreground star is readily detected both morphologically and via its anomalous line to continuum ratios, and rejected. As a selection criterion for HiI regions we specified that a feature must contain at least nine contiguous pixels, each with an intensity of at least three times the rms noise level of the local background. Any object not meeting this criterion was indistinguishable from noise and therefore treated as noise. The rms noise of the background-subtracted $\mathrm{H} \alpha$ image is 15 instrumental counts, the lower limits to the luminosity of the detected HiI regions, and to the radius of the smallest catalogued regions (the last two quantities are derived directly from the adopted selection criterion) are, respectively, $\log L_{\mathrm{H} \alpha}=37.65 \mathrm{erg} \mathrm{s}^{-1}$ and $\approx 75 \mathrm{pc}$.
In identifying and quantifying the parameters of the HII regions we had to overcome three complicating effects. Firstly, many HiI regions appear to overlap on the image. Without attempting to analyze what fraction of these overlaps implies real contact and what fraction is merely a projection effect, we adopted the solution proposed in Rand (1992) and followed in Knapen et al. (1993) and Rozas et al. (1996a) of counting each peak in $\mathrm{H} \alpha$ as representing a single HiI region. The flux of each HiI region was then estimated by integrating over the pixels which could be reasonably attributed to a given region. One will undoubtedly miss a number of HiI regions that are too weak to be detected in the vicinity of stronger emitters close by. This will influence the lower end of the LF but is not a significant factor in the determination of the shape of the true LF at the higher luminosity end (Rand 1992). Secondly, an HiI region is not necessarily circular. Thirdly, the presence of diffuse $\mathrm{H} \alpha$ may lead to ill-defined edges of Hil regions, introducing some systematic errors, above all 
for the weakest regions (see Sect. 9, and Zurita et al., in preparation). The detection and cataloguing of the HII regions were performed using a new program developed by one of the authors (Heller et al., in preparation).

The program, identifies each HiI region, measures the position of its centre, derives the area in pixels and the flux of each region, integrating all the pixels belonging to the region and subtracting the local background value. The background values were fixed before running the code. As the background was not constant across the frame, we selected 96 areas in the image with circular aperture of radius 7 pixels, so that an appropriate value determined from the nearest background area was applied to each HII region. As a result of test measurements, we found that the uncertainties in the HII region fluxes caused by the variation in the background lie between $\sim 10 \%$ for the faintest regions and $<1 \%$ for the brightest.

The code allows us to edit the catalogue by deleting, adding, separating or rounding (with a circular aperture) the regions by hand where the automatic process produces regions with faint wings of very irregular shape.

The number of catalogued HII regions in NGC 7479 is 1009, and for all the HiI regions we determined equatorial coordinate offsets from the nucleus and deprojected distances to the centre (in arcsec), using the inclination angles and position angles given by Laine \& Gottesman (1998) $\left(i=51^{\circ}, \mathrm{PA}=22^{\circ}\right)$. We also determined the diameter and the $\mathrm{H} \alpha$ luminosity (in $\mathrm{erg} \mathrm{s}^{-1}$ ) of each HiI region. The catalogues are available via the CDS, or from the authors. In Fig. 2 we show schematically the positions of the HII regions in the disc of NGC 7479, on a deprojected RA-dec grid centred on the nucleus of the galaxy.

\section{Luminosity functions}

The full count of HII regions seen in Fig. 2 is presented in differential form, as a log-log plot in bins of 0.15 dex, in Fig. 3. The luminosity range in $\mathrm{H} \alpha$ is limited at the high end by the luminosity of the brightest detected region, and at the low end by our criteria for a detection, and covers $\log L_{\mathrm{H} \alpha}=37.0$ to $\log L_{\mathrm{H} \alpha}=41.0$. The limit of completeness, however, is $\log L_{\mathrm{H} \alpha}=38.0$, so that the apparent broad peak in the distribution just below this luminosity is an artefact due to observational selection. The best single linear fit of the data to the points above $\log L_{\mathrm{H} \alpha}=38$, corresponding to a power law $\mathrm{d} N(L)=A L \alpha \mathrm{d} L$, has a gradient $-0.83 \pm 0.06$ (i.e. $\alpha=-1.83 \pm 0.06$ ). We have also carried out a bi-linear fit, based on the evidence that at $\log L \sim 38.8$ there is an apparent change in slope and the two slopes correspond to values of $\alpha=-1.33 \pm 0.04$ and $-1.99 \pm 0.07$. The correlation coefficients for the rms fits are, for the single linear case 0.969, and for the bi-linear case 0.979 and 0.978 respectively.

The LF for NGC 7479 is broadly similar to LF's of other spirals. As noted above, the total number of regions

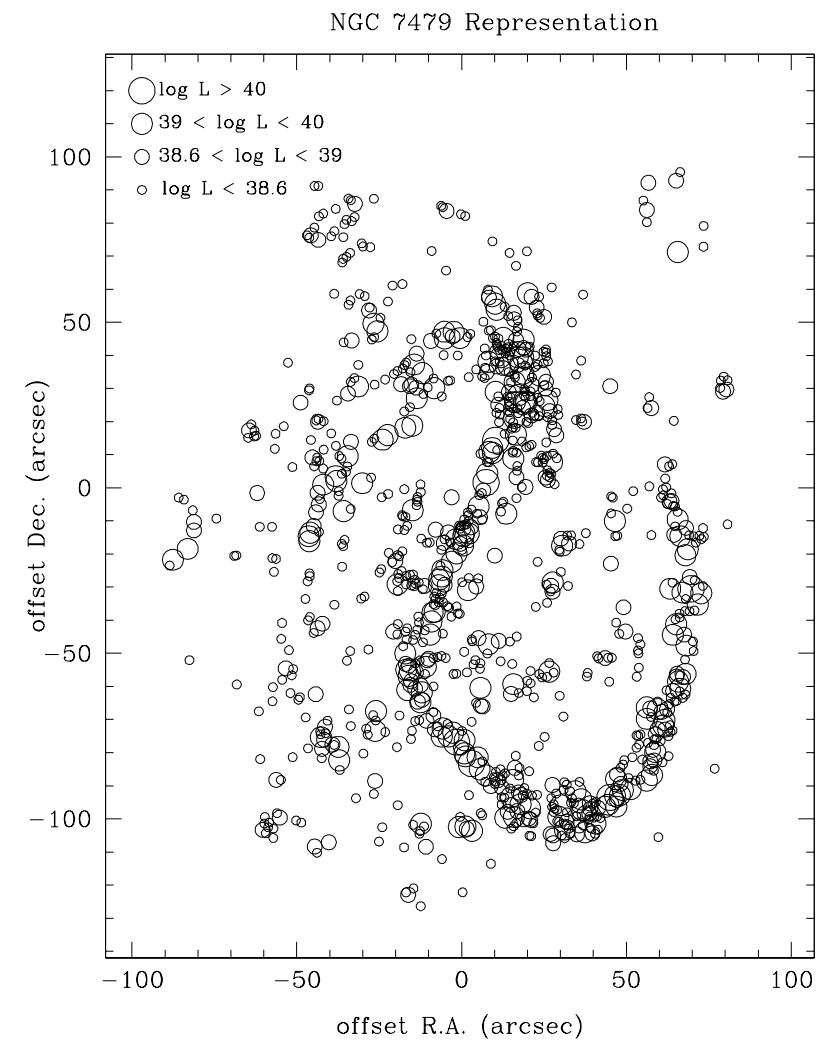

Fig. 2. Representation of the positions of the measured HII regions. Symbols show ranges of $\log L$. Coordinates of the centre of the image are $\mathrm{RA}=23^{\mathrm{h}} 4^{\mathrm{m}} 56.64^{\mathrm{s}}$ Dec $=12^{\circ} 19^{\prime} 22.9^{\prime \prime}$ (J2000)

catalogued is 1009. A total between $500-1000$ is typical of the number of distinguishable regions in galaxies with masses in the range of that of the Galaxy, at distances between 10 - 35 Mpc (Rozas et al. 1996a).

The criteria limiting the detection of a region at low luminosity cause the observational cut-off and give rise to the artificial peak in the LF just below $\log L_{\mathrm{H} \alpha}=38$. The true complete LF presumably rises with decreasing luminosity, but probably flattens off in the range below $\log L_{\mathrm{H} \alpha}=36.0$ as found by Walterbos et al. (1992) for M 31.

In previous work on other spiral galaxies (Rozas et al. 1996a) we found the change in slope in the LF and we explained it as a manifestation of a transition in the physical properties of the regions, but in these cases the luminosity of the transition was always $\log L_{\mathrm{Str}} \sim 38.6$ ( $\sim 0.2$ dex lower than in NGC 7479). As NGC 7479 is a barred galaxy with unusually strong star formation in the bar, we tested the hypothesis that the differences in the LF might be due to this intense star formation activity under somewhat different physical conditions from those in the disc. To perform this test we constructed separately the LF's for the HII regions of the bar and of the disc. The results are shown in Figs. 5 and 6 . The extent and structure of the bar were obtained from a bulge-disc-bar 


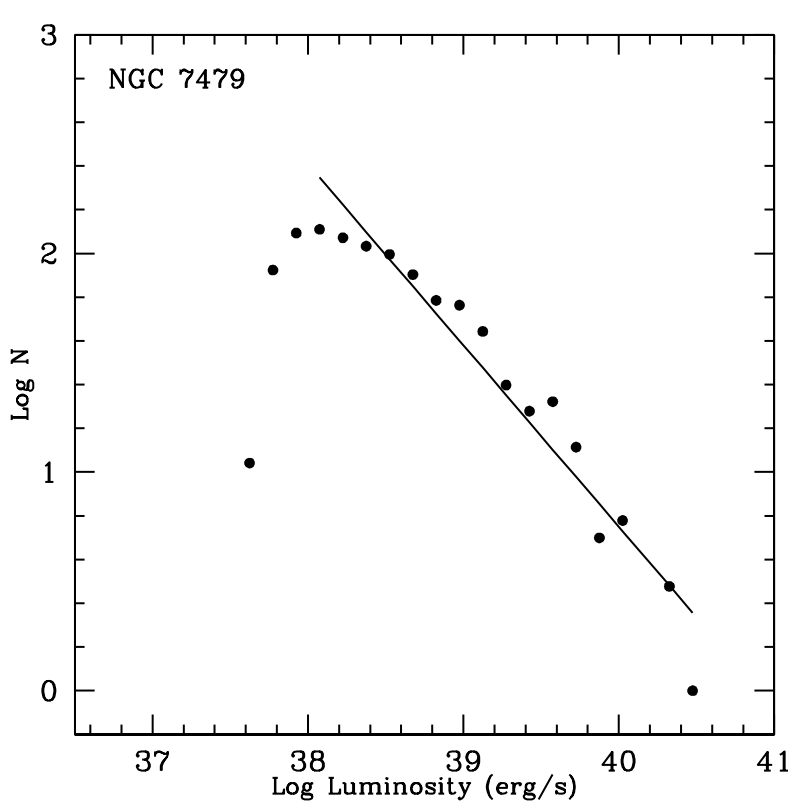

Fig. 3. Luminosity function for the complete sample of HII regions from the catalogue. The straight line indicates the best linear fit for luminosities greater than the completeness limit $\log L=38.0$

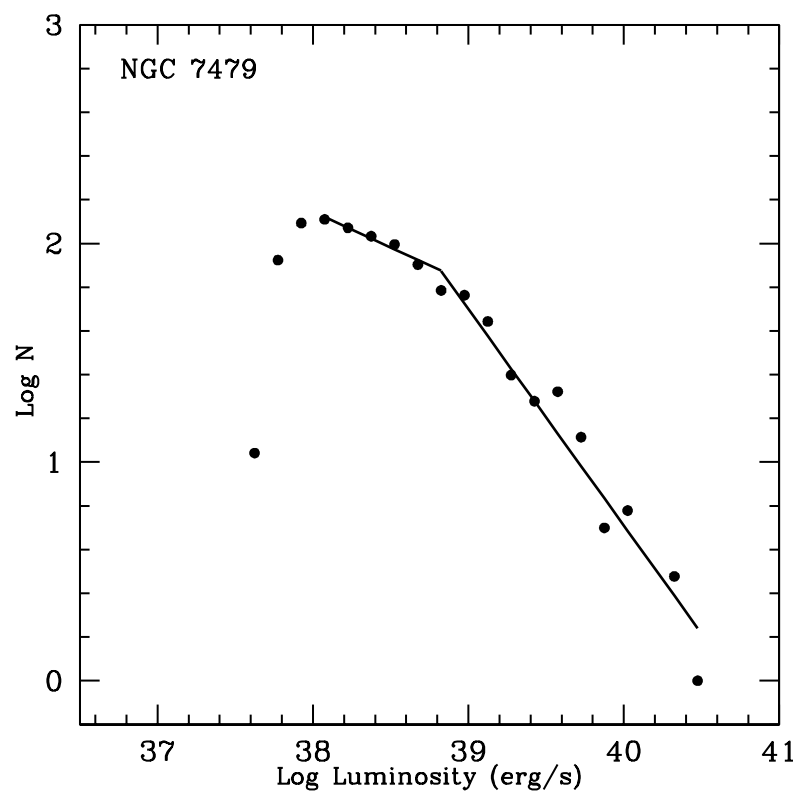

Fig. 4. Bi-linear fit for the luminosity function, based on previous experience with late-type spirals (Rozas et al. 1996a; Rand 1992; Knapen et al. 1993; Kennicutt et al. 1989). The plot has more scatter than those found for other spirals due to the irregularity of the LF of the HII regions in the bar (see Fig. 6)

decomposition of the surface brightness profiles along the major and minor semiaxes of the bar according to the decomposition method of Prieto et al. (1998). Using the fit obtained by this method, the length of the bar was $\sim 60$ " in the $I$ band. The LF for the 629 Hil regions detected in the disc is presented in Fig. 5. For this LF the change in slope is cleaner than for the total LF. The slopes for the bi-linear fit give $\alpha$ values of $-1.25 \pm 0.04$ and $-2.10 \pm 0.07$

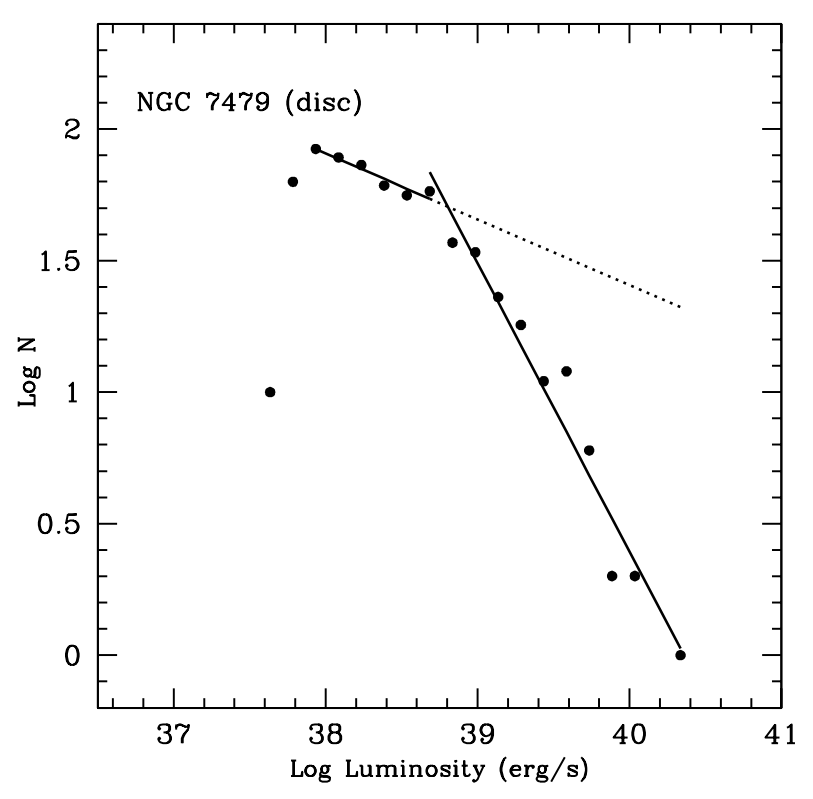

Fig. 5. Bi-linear fit for the luminosity function of the HiI regions of the disc. The change in slope is seen at $\log L=38.65$ $\left(\mathrm{erg} \mathrm{s}^{-1}\right)$ close to the values previously found for other spiral discs

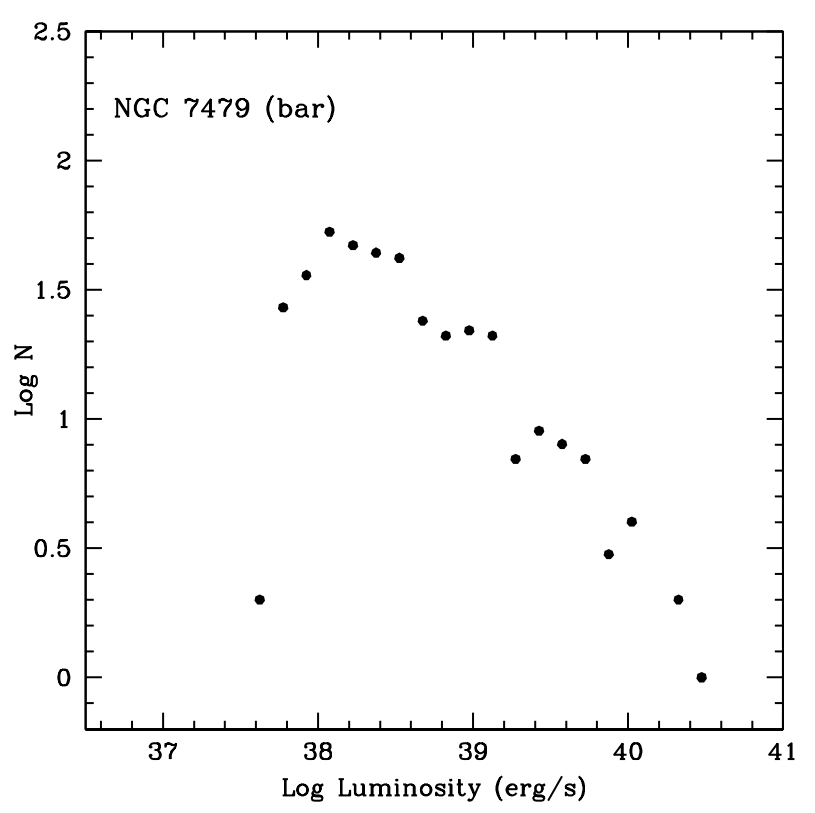

Fig. 6. Luminosity function of the HiI regions of the bar. The function is clearly less well-behaved than that for the disc

with correlation coefficients $r=0.956$ and 0.980 respectively, while in the best single linear fit $\alpha=-1.82 \pm 0.07$ and $r=0.958$. The change in slope for the HII regions in the LF of the disc occurs at $\log L=38.65 \pm 0.15$ which is in the range found for the galaxies we cited above. The change of slope, accompanied by a slight bump in the LF, has been detected in the seven galaxies so far examined in this degree of detail (Rozas et al. 1996b; Knapen et al. 1993; Cepa \& Beckman 1989, 1990). Adding the value for NGC 7479 to the set of values previously measured for 
other galaxies, we find that the rms scatter in $L_{\mathrm{Str}}$ in the full set of objects is 0.08 mag. This low scatter can be explained if the IMF at the high luminosity end of the mass function changes little from galaxy to galaxy, i.e. varies little with metallicity. For evidence on this point we can quote Massey et al. (1995), who show that the IMF slopes in the range of stellar masses above $10 M_{\odot}$ are the same for the Galaxy and for the LMC, whose metallicity is 10 times lower. Another necessary condition is that the rate of emission of ionizing photons from a young stellar cluster rises more rapidly than the mass of its placental cloud, a condition which we have examined (Beckman et al. 1999), and shown to hold where the appropriate observations exist.

If we look at Fig. 6, where the LF for the 380 Hir regions of the bar is presented, we can see clearly that the irregularity found in the total LF is due to the incorporation of the Hir regions of the bar in the total LF. The LF of the bar regions shows far greater departures from linearity in the log-log plane. The number of very luminous regions is especially high. Clearly star formation conditions in the bar differ from those in the disc.

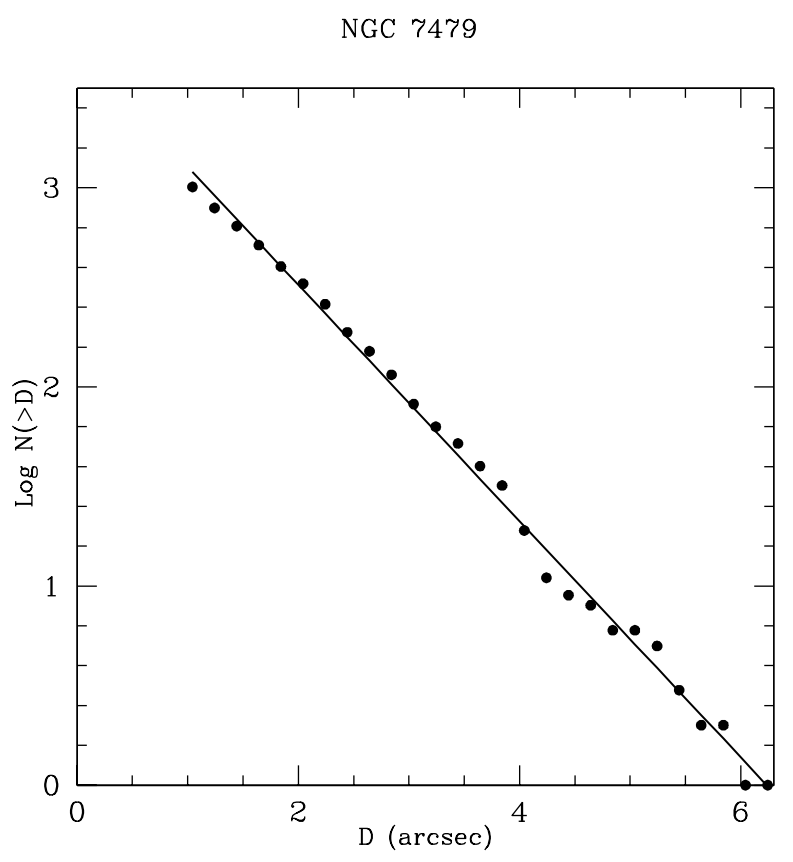

Fig. 7. Integral diameter distribution function of all HiI regions of NGC 7479. The straight line indicates the best fit

\section{Diameter distribution}

In Fig. 7 we present the integral diameter distribution: the number of regions with diameters greater than a given value as a function of diameter. In previous studies (van den Bergh 1981; Hodge 1987; Cepa \& Beckman
1989; Ye 1992; Knapen et al. 1993; Rozas et al. 1996b) it has been found that the diameter distribution of the HiI regions can be well fitted by an exponential of form $N(>D)=N_{0} \exp \left(-D / D_{0}\right)$ where $D_{0}$ is a characteristic diameter, and $N_{0}$ is an (extrapolated) characteristic value for the total number of regions. From the observations represented in Fig. 7 we obtain the values $N_{0}=5000 \pm 300$ and $D_{0}=110 \pm 2 \mathrm{pc}$ assuming $H_{0}=75 \mathrm{~km} \mathrm{~s}^{-1} \mathrm{Mpc}^{-1}$ (and $D_{0}=167 \pm 2$ for $H_{0}=50 \quad \mathrm{~km} \mathrm{~s}^{-1} \mathrm{Mpc}^{-1}$ ). We have given two values for $D_{0}$, the first being a more realistic estimate, while the second is included to compare explicitly with previously published values, where $50 \mathrm{~km} \mathrm{~s}^{-1} \mathrm{Mpc}^{-1}$ for $H_{0}$ has been used. The value for $N_{0}$ is within the range found for previously observed late type spirals, as given in the papers cited in the present paragraph. $D_{0}$ has the value predicted (within its error bars) for a galaxy of its measured absolute luminosity according to the observational plot of $D_{0}$ versus luminosity in Hodge (1987).

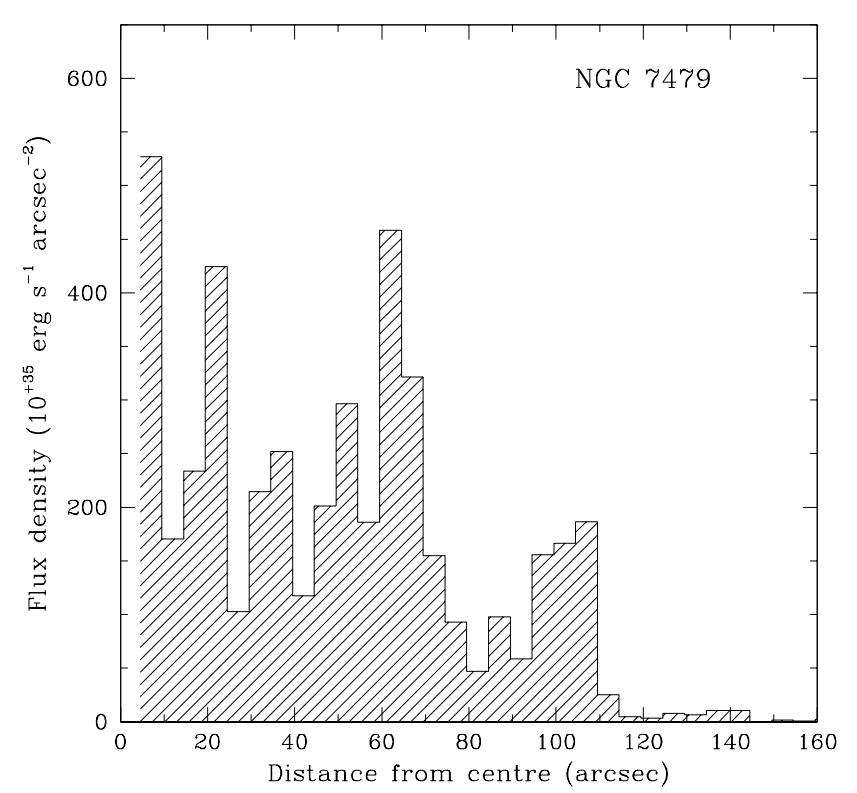

Fig. 8. Flux density distribution of all the HII regions of NGC 7479 as a function of the deprojected distance to the centre of the galaxy

\section{Flux and number density distributions}

In Figs. 8 and 9 we show the flux density distribution and the number density distribution, respectively, of the HII regions across the face of the galaxy; the two plots are on linear scales, both in ordinate, intensity, and in abscissa, distance from the centre. To do this we divided the disc into rings using the values of PA and $i$ of the galaxy 


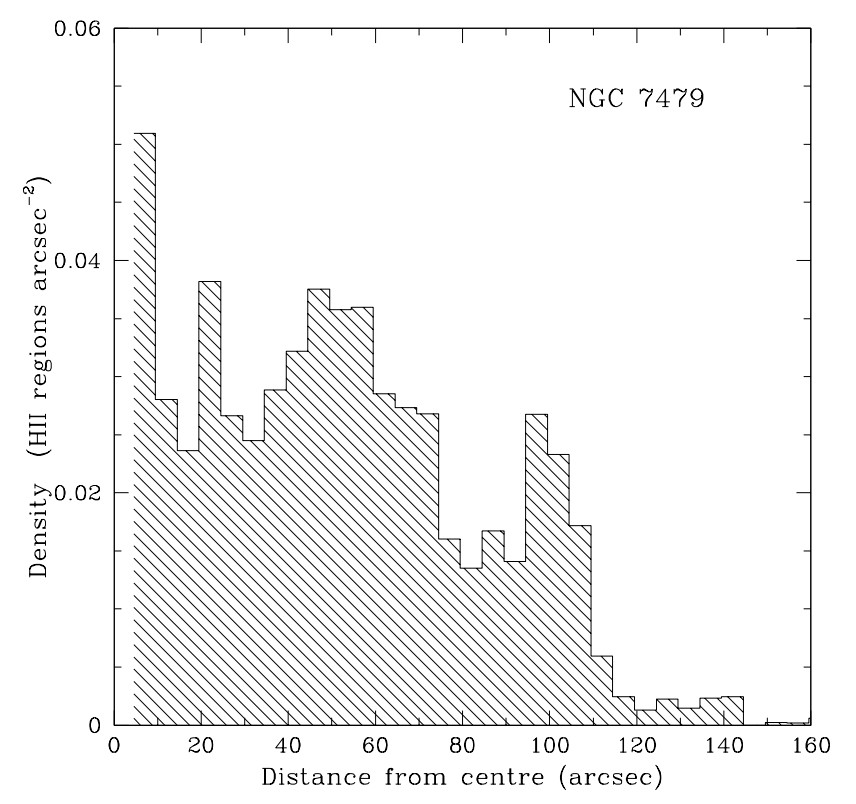

Fig. 9. Number-density distribution of all the HiI regions of NGC 7479 as a function of deprojected distance from the centre

(Table 1), but we have not shown the number density or the flux density in the inner ring, because the value in this small area was affected by this division. Two trends are clear in these plots: firstly the trend to lower flux, and number density, with increasing radius, which clearly reflects the standard radial decline in surface density of each major component of this disc galaxy; secondly the presence of notable peaks and troughs within this general behaviour. Two major peaks, at $5 \operatorname{arcsec}(770 \mathrm{pc})$ and at $60 \operatorname{arcsec}(9.3 \mathrm{kpc})$ from the centre are especially prominent. These represent the major star forming regions along the bar and at the end of the bar respectively, while the smaller peaks between $90-100 \operatorname{arcsec}(13.9-15.5 \mathrm{kpc})$ correspond to peaks in the star formation along the prominent southern spiral arm. The underlying radial flux density distribution can be fitted by an exponential of form

$f(r)=B \mathrm{e}^{-\left(r / h_{\mathrm{H} \alpha}\right)}$

from which we derive a value for the $\mathrm{H} \alpha$ scale length, $h_{\mathrm{H} \alpha}=2.4( \pm 0.3) \mathrm{kpc}$.

\section{Luminosity-volume relation}

Figure 10 shows the relation between the logarithmic $\mathrm{H} \alpha$ luminosity of a given HII region, $\log L$ (with $L$ in erg $\mathrm{s}^{-1}$ ), and the logarithm of the cube of the radius $\log r^{3}(r$ in $\operatorname{arcsec})$. If this relation were to maintain a constant slope, this would mean that the product of the electron density, the proton density and the filling factor (fraction of the volume filled with ionized medium,
Osterbrock 1974) were constant, independent of the luminosity of the HII regions. If regions of high luminosity were density bounded it would yield an increasing slope with increasing volume, i.e. the relation should show a concave upward curve, corresponding to an increase in the average filling factor. Although the scatter in Fig. 10 is large, there is an upward curvature at high luminosity, which is in agreement with the general scenario in which the most luminous His regions in spirals (and in all probability in irregulars, see Fuentes 1997) are density bounded (Beckman et al. 1999; Rozas et al. 1996a). The observed distribution is discretized along the abscissa in Fig. 10 because $r$ is calculated by counting the number of pixels within a region, dividing by $\pi$ and taking the square root, which leads to an obvious systematic scatter increasing fractionally to lower luminosities i.e. to smaller radii. There are further factors leading to uncertainty in the observed values in Fig. 10. These are: (a) faint regions are difficult to detect against the true diffuse background of $\mathrm{H} \alpha$ within the galaxy; this is especially so within the arms and the bar, though less of a problem in the interarm disc. (b) Overlap may occur especially in the arms and the bar, which may cause significant underestimation of the volume. In Fig. 10, we have plotted HiI regions of the bar and of the disc, using different symbols. There is no clear difference between the properties of the two sets of regions characterized by this plot.

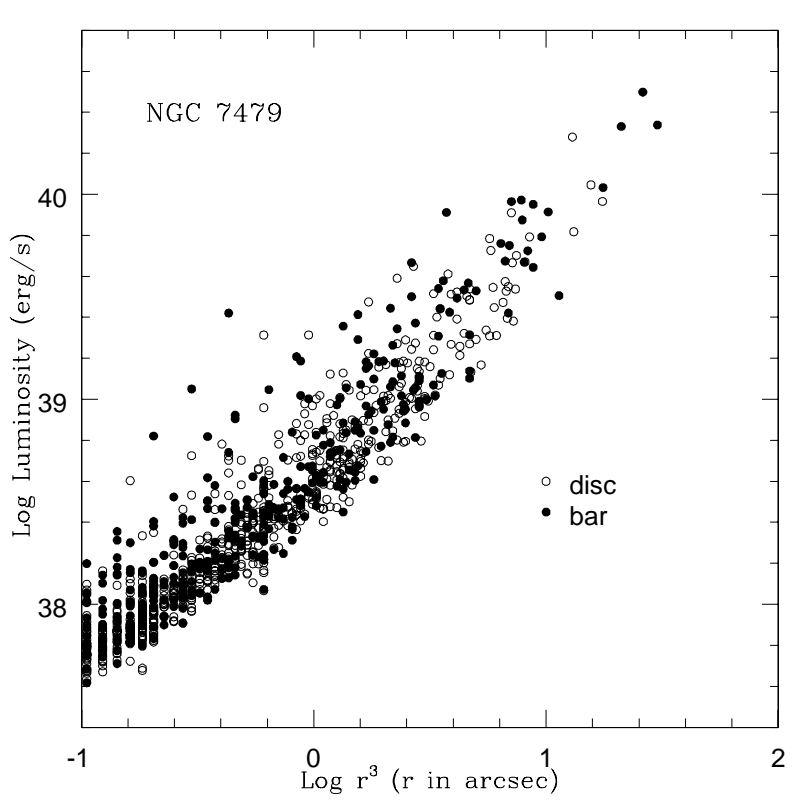

Fig. 10. Relation between the logarithm of the cube of the radius of an HiI region ( $r$ in arcsec) and the logarithm of its luminosity (in erg $\mathrm{s}^{-1}$ ). Fractional errors grow rapidly for log $r^{3}<-0.5$ due to the angular resolution limit in the image 
Table 2. Measured physical properties of individual HiI regions selected for their isolation and projected circularity: catalogue number, radius, luminosity, emission measure, rms electron density, filling factor, mass of ionized gas, log of the number of Lyman- $\alpha$ photons $\mathrm{s}^{-1}$ necessary to ionize this gas, equivalent number of O5V stars and an indication of the position (bar or disc) for each selected HII region

\begin{tabular}{|c|c|c|c|c|c|c|c|c|c|}
\hline $\mathrm{N}^{\mathrm{O}}$ & 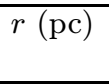 & $\begin{array}{c}\log L \\
\left(\operatorname{erg~s}^{-1}\right)\end{array}$ & $\begin{array}{c}\mathrm{Em} \\
\left(\mathrm{pc} \mathrm{cm} \mathrm{cm}^{-6}\right)\end{array}$ & $\begin{array}{c}<N_{\mathrm{e}}>_{\mathrm{rms}} \\
\left(\mathrm{cm}^{-3}\right)\end{array}$ & $\begin{array}{c}\text { filling factor } \\
\left(\times 10^{4}\right)\end{array}$ & $\begin{array}{c}\left(\mathrm{H}^{+}\right) / M_{\odot} \\
\left(\times 10^{-4}\right)\end{array}$ & $\overline{l o g} N_{\text {Lyc }}$ & $\bar{N}(\mathrm{O} 5 \mathrm{~V})$ & bar/disc \\
\hline 2. & 480 & 40.34 & 8300 & 4.1 & 9.4 & 150 & 52.21 & 313 & $\overline{\mathrm{B}}$ \\
\hline 8. & 370 & 39.96 & 6000 & 4.0 & 9.0 & 60 & 51.83 & 133 & $\mathrm{D}$ \\
\hline 10. & 320 & 39.95 & 7700 & 4.9 & 13.2 & 60 & 51.82 & 128 & B \\
\hline 11. & 340 & 39.91 & 6400 & 4.3 & 10.4 & 60 & 51.78 & 118 & B \\
\hline 13. & 300 & 39.91 & 8100 & 5.2 & 14.9 & 50 & 51.78 & 117 & $\mathrm{D}$ \\
\hline 16. & 330 & 39.79 & 5000 & 3.9 & 8.4 & 40 & 51.66 & 89 & B \\
\hline 17. & 320 & 39.79 & 5500 & 4.2 & 9.5 & 40 & 51.66 & 89 & $\mathrm{D}$ \\
\hline 26. & 310 & 39.67 & 4300 & 3.7 & 7.5 & 30 & 51.54 & 67 & B \\
\hline 37. & 280 & 39.55 & 3900 & 3.8 & 7.7 & 20 & 51.41 & 51 & $\mathrm{D}$ \\
\hline 39. & 260 & 39.54 & 4500 & 4.2 & 9.6 & 20 & 51.41 & 50 & $\mathrm{D}$ \\
\hline 50. & 250 & 39.49 & 4400 & 4.2 & 9.8 & 20 & 51.36 & 45 & B \\
\hline 59. & 240 & 39.43 & 4000 & 4.1 & 9.0 & 20 & 51.29 & 38.2 & B \\
\hline 75. & 230 & 39.31 & 3400 & 3.9 & 8.2 & 10 & 51.18 & 30 & $\mathrm{D}$ \\
\hline 81. & 210 & 39.29 & 3900 & 4.3 & 10.2 & 13 & 51.16 & 28.0 & $\mathrm{D}$ \\
\hline 94. & 200 & 39.19 & 3600 & 4.3 & 10.0 & 10 & 51.05 & 22.1 & B \\
\hline 103. & 210 & 39.18 & 3000 & 3.8 & 7.7 & 10 & 51.04 & 21.6 & $\mathrm{D}$ \\
\hline 121. & 220 & 39.10 & 2300 & 3.3 & 5.8 & 9 & 50.97 & 18.3 & $\mathrm{D}$ \\
\hline 143. & 220 & 39.04 & 2000 & 3.1 & 5.1 & 7 & 50.91 & 15.8 & $\mathrm{D}$ \\
\hline 152. & 223 & 39.01 & 1800 & 2.9 & 4.5 & 7 & 50.88 & 14.8 & $\mathrm{D}$ \\
\hline 168. & 190 & 38.99 & 2300 & 3.4 & 6.5 & 7 & 50.86 & 14.0 & B \\
\hline 208. & 200 & 38.88 & 1700 & 2.9 & 4.7 & 5 & 50.74 & 10.8 & B \\
\hline 273. & 170 & 38.72 & 1600 & 3.0 & 5.1 & 4 & 50.57 & 7.5 & $\mathrm{D}$ \\
\hline 323. & 160 & 38.62 & 1400 & 3.0 & 4.9 & 3 & 50.49 & 6.0 & $\mathrm{D}$ \\
\hline 327. & 160 & 38.61 & 1500 & 3.0 & 5.07 & 3 & 50.48 & 5.9 & B \\
\hline 391. & 140 & 38.51 & 1400 & 3.1 & 5.4 & 2 & 50.37 & 4.6 & $\mathrm{D}$ \\
\hline 399. & 130 & 38.49 & 1500 & 3.4 & 6.2 & 2 & 50.36 & 4.4 & B \\
\hline 477. & 120 & 38.40 & 1500 & 3.5 & 6.7 & 2 & 50.27 & 3.6 & $\mathrm{D}$ \\
\hline 511. & 130 & 38.33 & 1100 & 2.9 & 4.6 & 1.5 & 50.20 & 3.1 & B \\
\hline 660. & 80 & 38.15 & 1800 & 4.6 & 11.4 & 1.0 & 50.02 & 2.0 & B \\
\hline 752. & 90 & 38.05 & 1200 & 3.6 & 7.2 & 0.8 & 49.92 & 1.6 & B \\
\hline 818. & 90 & 37.97 & 1100 & 3.5 & 6.6 & 0.6 & 49.84 & 1.3 & B \\
\hline 828. & 90 & 37.95 & 900 & 3.2 & 5.7 & 0.6 & 49.82 & 1.29 & $\mathrm{D}$ \\
\hline 851. & 100 & 37.92 & 800 & 2.8 & 4.3 & 0.6 & 49.79 & 1.19 & $\mathrm{D}$ \\
\hline 913. & 80 & 37.85 & 900 & 3.2 & 5.7 & 0.5 & 49.72 & 1.02 & $\mathrm{D}$ \\
\hline 998. & 70 & 37.71 & 800 & 3.4 & 6.3 & 0.3 & 49.58 & 0.74 & $\mathrm{D}$ \\
\hline 997. & 80 & 37.71 & 700 & 2.9 & 4.7 & 0.3 & 49.54 & 0.74 & B \\
\hline 1001. & 70 & 37.69 & 800 & 3.3 & 6.0 & 0.3 & 49.55 & 0.70 & B \\
\hline 1009. & 70 & 37.62 & 700 & 3.1 & 5.1 & 0.3 & 49.49 & 0.60 & $\mathrm{D}$ \\
\hline
\end{tabular}

\section{Physical properties}

Using a distance to NGC 7479 of $31.92 \mathrm{Mpc}$ (derived assuming $H_{0}=75 \mathrm{~km} \mathrm{~s}^{-1} \mathrm{Mpc}^{-1}$ ) we employed the standard theoretical formula relating the surface brightness of an Hir region with its emission measure Em (Spitzer 1978), assuming that the recombination lines are formed under the conditions of case B (Osterbrock 1974), to calculate Em for each Hir region. A standard temperature of $10^{4} \mathrm{~K}$ was taken for the calculation. We performed this for a total of 39 regions (22 in the disc and the rest in the bar), covering the full range of observed radii. They were chosen as isolated, so that the uncertainties in calculating their luminosities due to the overlapping of other regions are never as high as $10 \%$. The results are plotted in Fig. 11. Values are given in Table 2 where the luminosity, radius, and emission measure are shown, as well as the electron density, computed from Em; the rms electron densities, derived from the emission measures, are plotted against radius of the HII region, in Fig. 12.

In Fig. 13 we show the rms electron densities $<N_{\mathrm{e}}>_{\text {rms }}$ for the same regions, plotted against luminosity. The general ranges and behaviour of Em and 


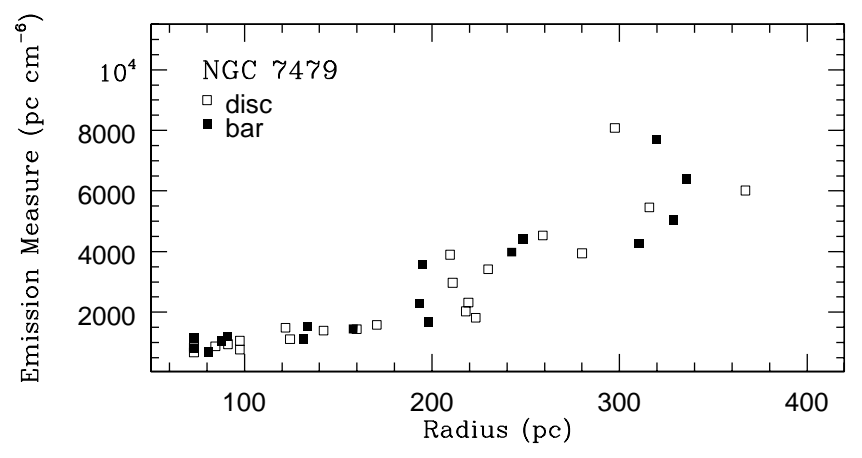

Fig. 11. Emission measure versus radius for the selected HII regions in NGC 7479

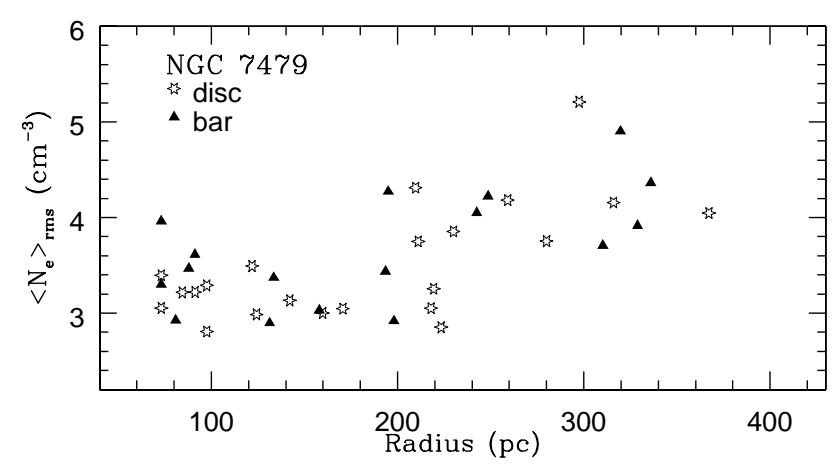

Fig. 12. rms electron density versus radius for the selected HII regions

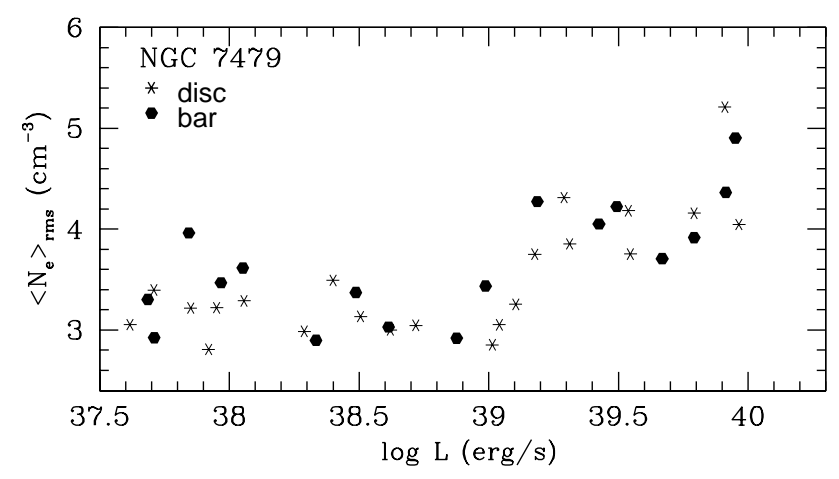

Fig. 13. rms electron density for the selected Hiı regions versus $\log L$
$<N_{\mathrm{e}}>_{\text {rms }}$ for NGC 7479 agree well with those found by Kennicutt (1984) and by ourselves (Rozas et al. 1996b) for extragalactic HiI regions. Due to observational selection, these tend to be more luminous and larger than Galactic regions.

Kennicutt (1984) first showed that the electron densities in the largest HII regions are of order $1 \mathrm{~cm}^{-3}$, which is comparable with that of the general diffuse interstellar medium. This is not unexpected, as the powerful central sources can ionize very large volumes of space, whose average matter density is not high. The measured values of $\left.<N_{\mathrm{e}}\right\rangle_{\text {rms }}$ vary by a factor two for the whole set of regions measured in NGC 7479; although the scatter within this range is high, there is a clear trend for $\left\langle N_{\mathrm{e}}\right\rangle_{\text {rms }}$ to increase with $L$ for high luminosities. This density increase is consistent with the increase in surface brightness found for regions with $L>10^{38.6} \mathrm{erg} \mathrm{s}^{-1}$, associated with the change of regime from ionization bounding to density bounding hypothesised in Rozas et al. (1996b), in Beckman et al. (1999), and in Rozas et al. (1998).

To infer the uncertainties in the calculation of the Em and $\left\langle N_{\mathrm{e}}\right\rangle_{\text {rms }}$, we have estimated the propagation of the error in the determination of the radius and in the flux of the regions. Although most of the regions in the catalogue are not perfect spheres and, in general, it is not easy to estimate the uncertainties in the determination of the radius, this is not the case for the regions selected in the sample; they are nearly spherical since they have been chosen for their circularity in projection. Errors in the determination of the radius are $\sim 0.5$ pix, and the uncertainty in the determination of the flux is of the order of the flux in an external ring of the region with width 0.5 pix and radius equal to that of the region.

In this way, the relative uncertainty in the calculation of the Em is of the order of $50 \%$ for smallest HiI regions ( $\log L<38$, below the completeness limit of the LF) and $\sim 10 \%$ in more luminous regions. For $\left.<N_{\mathrm{e}}\right\rangle_{\text {rms }}$ the resulting uncertainty is between $40-50 \%$ for faint regions and decreases to well below $10 \%$ as the luminosity reaches values typical of brighter regions. In order to calculate the filling factor we need to know, as well as the value of the rms electron density $\left\langle N_{\mathrm{e}}\right\rangle_{\text {rms }}$ values of the in situ electron density $N_{\mathrm{e}}$ for each region. We have not measured these values for NGC 7479, but have used a "canonical" mean value of $135 \mathrm{~cm}^{-3}$ obtained by Zaritzky et al. (1994) for 42 HII regions in a large sample of galaxies, via the intensity ratio of the forbidden SiI doublet $\lambda \lambda 6717$, $6731 \AA$. The value of $N_{\mathrm{e}}$ might well differ from bar to disc and it would certainly be worth making direct spectroscopic comparison in NGC 7479. However for the present we cannot improve on the use of a constant value for all HiI regions.

The implicit model is that an HII region is internally clumpy, so that the observed flux comes from a high density component, which occupies a fraction $\delta$ (filling factor) of the total volume; the rest of the volume is filled with low 
density gas which makes a negligible contribution to the observed emission line strengths. The filling factors, computed from $\left(<N_{\mathrm{e}}>_{\mathrm{rms}} / N_{\mathrm{e}}\right)^{2}$ for the regions range from $4.310^{-4}$ to $1.510^{-3}$, a range which coincides well with those found for 5 galaxies in Rozas et al. (1996b). Values of $\left\langle N_{\mathrm{e}}\right\rangle_{\text {rms }}$ can also be used to estimate the mass of ionized gas, by integrating over the measured volume of the region, and multiplying by the mass of a hydrogen atom, using the formula:

$M\left(\mathrm{H}^{+}\right)=\int \delta^{1 / 2}<N_{\mathrm{e}}>_{\mathrm{rms}} m(p) \mathrm{d} V$.

Results are given in Table 2 for the selection of regions; the masses range from some $3000 M_{\odot}$ to $1.510^{6} M_{\odot}$. In Table 3 we also give the rate of emission of Lyman continuum photons required to maintain the regions ionized, assuming a case B regime. It is important to note here that the Lyman continuum luminosity of those most luminous regions which are density limited will in fact be considerably higher than that estimated directly via their $\mathrm{H} \alpha$ fluxes. For regions with $\log L_{\mathrm{H} \alpha} \geq 39$, the escaping flux is in fact greater than the flux trapped within the region and observed via $\mathrm{H} \alpha$. Finally we have used the estimates of Vacca et al. (1996) of the Lyc luminosity of stars as a function of their spectral type, to compute the equivalent number of $\mathrm{O} 5 \mathrm{~V}$ stars (emitting Lyc at a rate of $510^{49}$ photons/s) required to supply the luminosities of the regions listed in Table 3 .

\section{Number-luminosity-diameter relation}

In Fig. 14 we give a three-dimensional plot of the number of HII regions of the disc as a function of two separate parameters: luminosity and diameter. It is of use to present the data this way as it allows us to see any correlation between the variables, which may give a clue to the underlying physical properties of the regions. Above the 3-d plot we have a projection into the luminosity-diameter plane, on a grey scale, in which we can readily see a change in the slope at a specific luminosity, which shows up clearly in spite of the obvious wide scatter of diameters at a given luminosity. This change is the equivalent of a change in the internal surface brightness gradients of the individual regions, and we will examine this quantitatively below. We have attributed this change, found in other disc galaxies, (see Rozas et al. 1996; Beckman et al. 1999) to the "Strömgren" transition from ionization bounded regions to density bounded regions. The luminosity at which this occurs will be the same as that of the small glitch peak in the luminosity function, but is not as well defined in the luminosity-diameter plot since the peak in the LF is determined uniquely by the luminosity, while the change in slope of the luminosity-diameter plot has to be found amid the scatter of diameters in placental clouds with a range of intrinsic gas densities, giving rise to the spread seen in the grey scale projection.
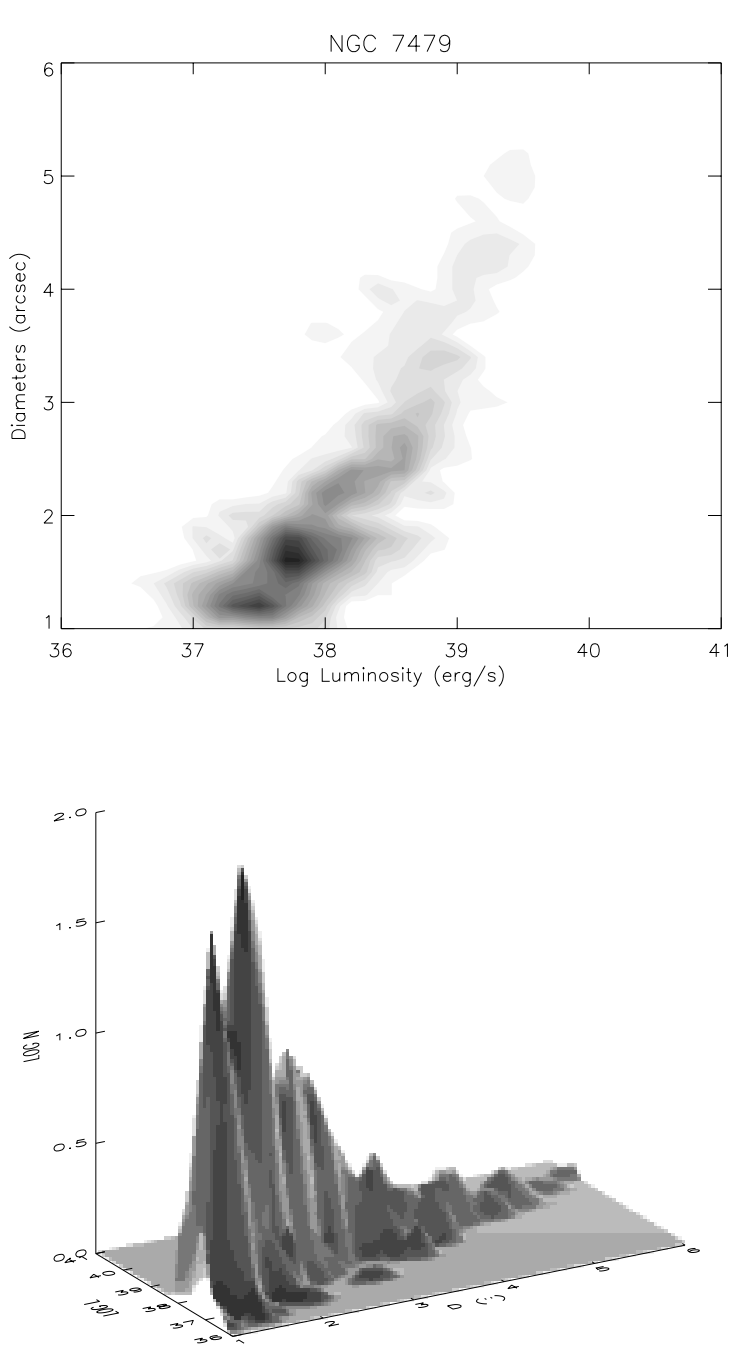

Fig. 14. Lower: 3-dimensional plot for the HII regions of the disc of NGC 7479 of the number (z-axis) of His regions as a function of luminosity ( $y$-axis) and diameter ( $x$-axis). Upper: Projection of the lower plot onto the diameter-log luminosity plane

\section{The diffuse $\mathrm{H} \alpha$ flux}

In this section we examine, using our data on NGC 7479 , whether the rate of escape of ionizing photons from HII regions, notably from the high luminosity regions which are density bounded, is sufficient to account for the ionization of the diffuse interstellar medium (ISM) outside the regions, detected via its $\mathrm{H} \alpha$ emission. In NGC 7479 the flux from the diffuse ionized gas (DIG) is a rather high fraction of the $\mathrm{H} \alpha$ emission, between $35 \%$ and $60 \%$ of the total. In order to make the test we needed to measure the diffuse $\mathrm{H} \alpha$ flux, compute the escaping ionizing flux from the Hil regions, and compare the two. If the second is the larger, we can conclude that the diffuse flux does not necessarily have an origin in processes other than ionization by the photons leaking out of HiI regions. If we can show 
a geometrical correlation between the computed escaping flux and the measured diffuse flux then we can take our conclusions a step further, and attribute the latter to the effects of the former.

\subsection{The total luminosity, and the diffuse luminosity: Observational}

To estimate the total luminosity emitted in $\mathrm{H} \alpha$ by NGC 7479 we first integrated the continuum-subtracted flux-calibrated $\mathrm{H} \alpha$ image over an elliptical area with the appropriate inclination and position angles, then subtracted off a constant level estimated for the background sky, integrated over this ellipse. The result is $L_{\mathrm{H} \alpha}($ total $)=(1.3 \pm 0.2) 10^{42} \mathrm{erg} \mathrm{s}^{-1}$, corresponding to

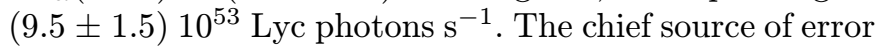
here is that due to the uncertainty in our determination of the sky level. Comparing this luminosity with the major sample of spirals in Devereux \& Young (1991), we find that it is just above the upper limit of the full range of this sample, i.e. NGC 7479 has a very high total $\mathrm{H} \alpha$ luminosity for a normal galaxy. To then estimate the diffuse flux we took more than one approach, due to a degree of geometrical uncertainty associated with the distribution of the diffuse emitting gas in the direction perpendicular to the plane of the galaxy. In the first method we used our catalogue of HiI region fluxes, integrated over the whole set, and subtracted this from $L_{\mathrm{H} \alpha}$ (total). The implied scenario here is that above an HII region there is much less diffuse gas in the total emitting column above the galaxy disc, so that to a first approximation this diffuse emission may be set to zero. In the second method, we used the equivalent areas of the HiI regions in the catalogue to prepare a masked image, in which the $\mathrm{H} \alpha$ surface brightness was first set to zero in the areas occupied by all the regions. Then before integrating over the resulting image to obtain the total diffuse flux, we could assign a constant, non-zero level to the masked areas, with the idea that even above an HiI region there is still some diffuse emission. One simple approach is to fill in the blanks to a surface brightness level equivalent to the mean value of the diffuse brightness measured in the field surrounding each region. This has the merit of being simple, but just as the use of blanks in the first method must give a lower limit to the total diffuse flux, filling these blanks fully at the level of the surrounding flux as in the second method will give an upper limit. The previous study by Ferguson et al. (1996) took the second approach, and the fractional $\mathrm{H} \alpha$ fluxes in the galaxies measured by these authors should be taken as upper limits, though not extremely far from true estimates. An intermediate approach is to fill the blanks with flux at a level which is the average for the diffuse flux over the whole galaxy. This again has the virtue of simplicity, and will give a value for the flux between the two limits, but is not guaranteed to give a precise value as a final result. To obtain the lower limiting diffuse flux we first obtained, from the full HII region catalogue, the fluxes and positions of all the regions with fluxes above the completeness limit, and integrated the flux of all these regions. To estimate the total due to weaker regions, we extrapolated the $\mathrm{LF}$ below $L_{\mathrm{H} \alpha}=10^{38} \mathrm{erg} \mathrm{s}^{-1}$, normalizing to the number in NGC 7479 at this luminosity, and following the curve measured by Walterbos \& Braun (1992) for M 31 at lower luminosities, down to their measurement limit of $10^{35} \mathrm{erg} \mathrm{s}^{-1}$. We were forced to this approach since M 31 is the only external galaxy for which complete measurements significantly below $10^{37} \mathrm{erg} \mathrm{s}^{-1}$ have been taken. The sum of the integrated flux due to these weaker regions, and that of the stronger regions was then subtracted from the total estimated for the galaxy. Then making a minor correction $(<2 \%$ of the total) for foreground stellar images and removing the sky level, we can find the diffuse flux (lower limit) by subtracting the HII region total from the galaxy total. The result found here was

$L_{\mathrm{H} \alpha}(\mathrm{DIG})_{1}=(4.6 \pm 1.5) 10^{41} \mathrm{erg} \mathrm{s}^{-1}$.

This can be used to compute an estimated requirement for the rate of Lyc photons flowing into the diffuse medium, which we did assuming case B, and a mean temperature in an HII region of $10^{4} \mathrm{~K}$, yielding

$L_{\mathrm{Lyc}_{1}}=(3.4 \pm 1.1) 10^{53}$ Lyc photons s ${ }^{-1}$.

In Fig. 15, we show the resulting aspect of the emission in $\mathrm{H} \alpha$ of the galaxy after subtracting off the catalogued HiI regions.

The upper limiting case was obtained by removing the HiI regions and filling the spaces at a level computed by taking a 4-pixel-wide ring around each region, and averaging the counts per pixel in this ring. Then, we integrated the masked image over the elliptical area we described in the determination of the total flux. After, we took off the difference between the luminosity from the HiI regions with $\log L<38$ using the M 31 luminosity function as described above, and the luminosity due to those regions with L less than $10^{38} \mathrm{erg} \mathrm{s}^{-1}$ which had been catalogued individually in our full HII region catalogue. This was done in order not to subtract any region twice.

Removing the sky level within the DIG area i.e. integration ellipse of the disc minus the Hit regions area, we found a value of

$L_{\mathrm{H} \alpha}(\mathrm{DIG})_{2}=(8 \pm 2) 10^{41} \mathrm{erg} \mathrm{s}^{-1}$,

which converts to

$L_{\mathrm{Lyc}_{2}}=(5.7 \pm 1.2) 10^{53}$ Lyc photons s ${ }^{-1}$,

for the upper limit. The intermediate case, which can be taken as the best estimate for the DIG component, was obtained by filling the HiI region blanks with the mean background taken over the whole disk. This is certainly a better approximation than either of the limiting cases, and yielded a value of

$L_{\mathrm{H} \alpha}(\mathrm{DIG})_{3}=(6 \pm 2) 10^{41} \mathrm{erg} \mathrm{s}^{-1}$,

which is produced by

$L_{\mathrm{Lyc}_{3}}=(4.5 \pm 1.2) 10^{53}$ Lyc photons s ${ }^{-1}$. 


\subsection{The ionizing flux escaping from the HII regions}

We can obtain an estimate of the escaping flux from regions of the disc on the basis of the assumption that the luminosity $\log L_{\mathrm{H} \alpha}=38.6 \mathrm{erg} \mathrm{s}^{-1}$ (i.e. the Strömgren luminosity, $\left.L_{\mathrm{Str}}\right)$ marks the boundary between the population of ionization bounded and density bounded HII regions. This is a simplifying assumption, because there will be some leak-out of photons from the less luminous regions, and some fluctuation in the degree of escape from the more luminous, but it allows us to make a first order estimate, within the theoretical framework given in Beckman et al. (1999). Using this, we can extrapolate the LF for NGC 7479, with its measured slope for the range below $L=L_{\mathrm{Str}}$, to give the predicted LF if all the Lyc photons produced with the HII regions had been downconverted to $\mathrm{H} \alpha$ inside them. By then subtracting off the measured LF for the regions observed with $L>L_{\mathrm{Str}}$, and integrating this difference using the maximum observed luminosity of a region as our upper limit, we obtain an estimate of the escaping flux. The first simple test to apply is whether this luminosity is as big as the observed $\mathrm{H} \alpha$ luminosity in the diffuse emission (or at least of the same order, since we are taking into account only the disc HII regions). If not, our basic hypothesis has been shown to be inadequate, but if there are sufficient escaping photons then we have at least shown that escaping photons from the density bounded regions could be the originators of the diffuse emission. This would be a sufficient though not a necessary condition that the ionization of the diffuse medium is caused by these escaping photons.

The result of this estimate of the escaping flux, $L_{\mathrm{esc}}$, for NGC 7479, using the LF in Fig. 5 is

$L_{\text {esc(disc) }}=2.210^{42} \mathrm{erg} \mathrm{s}^{-1}$ in $\mathrm{H} \alpha$, which corresponds to $1.610^{54}$ Lyc photons $\mathrm{s}^{-1}$.

We may consider this value as a lower limit to the escaping flux that is, in principle, available to ionize the diffuse gas, since there must be a considerable photon flux escaping from the regions of the bar. The problem is that due to the irregularity of the LF of the bar we cannot apply the above method, based on the linearity of the LF, to estimate escaping flux from the density bounded regions of the bar. However, the escaping flux in the disc alone is sufficient to ionize the diffuse medium of NGC 7479 (neglecting in this first approximation the necessity of analyzing carefully the geometrical problem of the DIG and the location of the ionizing photon sources) since the escaping disc flux is higher than the Lyc photon flux required to ionize the total DIG of the galaxy.

One rough approximation to calculate the escaping flux from the bar regions is to consider that the escaping flux in a density bounded region of the bar is a determined fraction of its luminosity in $\mathrm{H} \alpha$. For the HII regions of the disc, using the method described above, this escaping flux, for a region with observed flux $L_{\mathrm{H} \alpha}$, varies between $1.5 L_{\mathrm{H} \alpha}$ and $10.0 L_{\mathrm{H} \alpha}$, with a mean value of

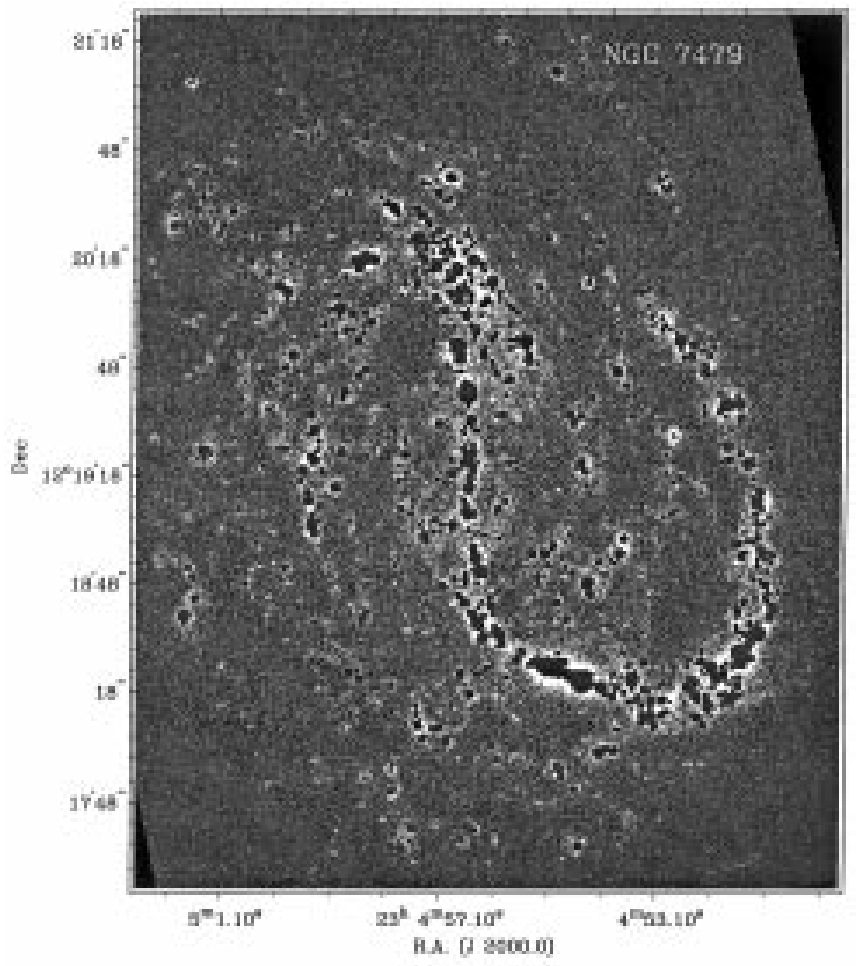

Fig. 15. Diffuse emission in $\mathrm{H} \alpha$ found after subtracting off the catalogued His regions (these appear as uniform regular filled grey circles). The close geometrical association of the diffuse $\mathrm{H} \alpha$ with above all, the most luminous HiI regions is evident

$3.5 L_{\mathrm{H} \alpha}$. Extrapolating this result to the regions of the bar with $\log L>38.6$ we found that

$L($ bar $)=6.010^{41} \mathrm{erg} \mathrm{s}^{-1}=4.410^{53}$ Lyc photons s $^{-1}$

so for the whole galaxy

$L_{\text {esc }} \simeq 2.810^{42} \mathrm{erg} \mathrm{s}^{-1}=2.010^{54}$ Lyc photons s$^{-1}$.

Even if we are overestimating this escaping flux, the fact that our initial estimate is bigger by a factor of over 4 than the flux needed to ionize the diffuse medium suggests strongly that in NGC 7479 there are prima facie grounds for our hypothesis to be supported.

The geometrical correlation between the positions of the density bounded regions and the observed diffuse $\mathrm{H} \alpha$ is clear in Fig. 15, showing that evidence for a causal link is present. This evidence was pointed out by Ferguson et al. (1996) in the galaxies they studied but without distinguishing between density and ionization bounded HII regions.

In order to proceed further with tests for the scenario, it will be necessary to make detailed models in which the degree of clumping in the diffuse medium can be realistically simulated, to see whether the mean free path can be long enough for the photons from the HiI regions to cause the geometrical distribution of $\mathrm{H} \alpha$ observed. We would also need to examine more carefully the details of this distribution as observed in a number of discs, to be able 
to model its dependence on the positions of the density bounded Hir regions as the suspected principal sources of its ionization (Zurita et al. 1999, in preparation).

By subtracting from the total hypothetical flux escaping from the Hil regions the value of the measured diffuse flux, we obtain an estimate of the Lyc flux which escapes completely from NGC 7479 . This value is $\geq 1.410^{54}$ photons $\mathrm{s}^{-1}$.

\section{Conclusions}

The main results of the present paper, where we analyze the statistics and properties of HiI regions in the disc of NGC 7479 are summarized below.

1. Using a high quality continuum-subtracted $\mathrm{H} \alpha$ image of the grand-design spiral NGC 7479, we have catalogued a total of 1009 HII regions. The catalogue includes positions, radii and $\mathrm{H} \alpha$ fluxes of all HII regions. Tables containing all these data are available through CDS or directly from the authors.

2. The slope of the LF agrees broadly with slopes for other galaxies of comparable morphological types.

3. We have found a change in slope in the LF of the HiI regions of NGC 7479 that occurs at a luminosity slightly higher $(\simeq 0.2 \mathrm{dex})$ than that found in other galaxies of the same morphological type. Due to the intense star formation in the bar of NGC 7479, we decided to construct separately the LF's for the HII regions of the bar and of the disc, finding that the LF of the disc is in no way different from that found in previous papers for galaxies of the same morphological type. The anomaly in the global LF is thus due to different star formation conditions in the bar. This must be due to the effects of gas dynamical parameters on the stellar IMF, and the physical conditions in the clouds, as we do not find significant differences between the physical properties of the Hil regions of the bar and of the disc.

4. The integrated distribution function of the HII region diameters can be well fitted by a exponential function. The value of the characteristic diameter lies within the range reported previously in the literature for galaxies of similar morphological types.

5. The characteristic scale size of the HII regions of a galaxy depends on the absolute luminosity of the galaxy; it is larger for more luminous galaxies (Hodge 1987). The result for NGC 7479 agrees with the fit presented by Hodge.

6. The dependence of $\log L$ on the volume $\left(r^{3}\right)$ for the regions in NGC 7479 lies close to a straight line, with a slope near unity. There is a tendency for the relationship to show curvature, corresponding to the onset of density bounding at high luminosities, but the dispersion in luminosity at a given volume is such that using only this plot we cannot draw a clear physical inference on this point.
7. The densities, filling factors, masses, and ionization indices derived from the luminosities and sizes of a selected set of representative regions, through the range of observed luminosities for NGC 7479, are in agreement with those found in the previous literature on extragalactic HiI regions.

8. The physical properties encountered cover a wide range, but all have moderately low electron densities, always much less than $10 \mathrm{~cm}^{-3}$, of the order of mean interstellar number densities in galaxies. These values, differ from the values found for the central densities of HiI regions (Rozas et al. 1998) by two orders of magnitude, but if we assume gaussian internal density distributions and weighting the densities as shown in Table 2 of Rozas et al. (1998), by volume, using the diameters determined here, we find mean electron densities within $20 \%$ of the values found here.

9. In the $3 \mathrm{D}$ diagram of number-luminosity-size, and in the projection on the luminosity-radius plane, we detect curvature in the high luminosity range, which would be predicted for the transition between ionization-bounded and density-bounded regions; this is only hinted at in the luminosity-volume graph. The characteristic mass scale for the onset of the curvature, or the change of slope shows the limit of the masses of the clouds of neutral gas which give rise to stellar associations (Rozas et al. 1996b; Beckman et al. 1999; Rozas et al. 1998). The luminosities which are associated with these changes in slope coincide in value in all galaxies studied by us, and also in those few galaxies subject to a similar study for which there exist data of equivalent quality: NGC 157, NGC 3631, NGC 6764, and NGC 6951 (Rozas et al. 1996a), M 51 (Rand 1992), NGC 6814 (Knapen et al. 1993), NGC 4321 (Knapen 1998 , in preparation). The value of this critical luminosity is $\log L=38.6 \pm 0.15 \mathrm{erg} \mathrm{s}^{-1}$, (for an assumed value for $H_{0}$ of $75 \mathrm{~km} \mathrm{~s}^{-1} \mathrm{Mpc}^{-1}$ ), and it coincides with the luminosity at which there is a "glitch", i.e. a jump, and/or a change of slope, in the luminosity functions, as reported in Rozas et al. (1996a) and previous papers (e.g. Kennicutt et al. 1989; Rand 1992; Knapen et al. 1993).

10. The total flux emitted in $\mathrm{H} \alpha$ by $\mathrm{NGC} 7479$ is $L_{\mathrm{H} \alpha}($ total $)=(1.3 \pm 0.2) 10^{42} \mathrm{erg} \mathrm{s}^{-1}$, which indicates that NGC 7479 is a high luminosity galaxy in $\mathrm{H} \alpha$. Its luminosity is over three times higher than the total $\mathrm{H} \alpha$ luminosity for the most luminous galaxy of the sample of grand design galaxies cited above (NGC 157, NGC 3631, NGC 6951, NGC 6764), and higher too than the total $\mathrm{H} \alpha$ luminosity of NGC 247 and NGC 7793, studied by Ferguson et al. (1996). This is due principally to the intense star formation in the very strong bar that characterizes this galaxy.

11. The diffuse flux from the ISM of NGC 7479 has been calculated by three methods to bracket the observational uncertainty inherent in observing any galaxy 
projected in a plane and due to crowding effects. We have found that the diffuse flux in $\mathrm{H} \alpha$ is a high fraction, between $35 \%-60 \%$ of $L_{\mathrm{H} \alpha}$ (total). This result is similar to the ionized gas fraction found by Ferguson et al. (1996) in NGC 247 and NGC 7793 (30\% - 50\% of the total $\mathrm{H} \alpha$ emission).

12. With the assumption that the most luminous HII regions, those with $L>10^{38.6}$ erg $\mathrm{s}^{-1}$, are density bounded, they are likely to be the main ionizing sources of the interstellar medium, since:

(a) The calculated leak-out of Lyc photons from these regions is enough to ionize the diffuse medium in this galaxy (in fact it exceedes the $L_{\mathrm{H} \alpha}(\mathrm{DIG})$ by a factor of over 2), even if we take into account only the leak-out of Lyc photons from regions of the disc. This excess is especially important here since a significant fraction of these photons will not be absorbed within the galaxy and must escape into the intergalactic medium.

(b) There is a clear geometrical correlation between the observed diffuse $\mathrm{H} \alpha$ flux and the positions of the density bounded Hil regions.

This result will be followed up with detailed models to include the degree of clumping in the diffuse medium (Zurita et al. 1999) in order to further investigate whether the diffuse $\mathrm{H} \alpha$ is caused by escaping Lyman photons from, especially, the most luminous HiI regions.

13. On the basis of our hypothesis of density bounding the calculated ionizing flux which escapes completely from the galaxy without being trapped in the diffuse component is $\geq 1.410^{54}$ photons $\mathrm{s}^{-1}$. If this were generalizable for spirals and irregulars, it would have important consequences for the ionization of the intergalactic medium (see also Zurita et al. 1999).

Acknowledgements. The William Herschel Telescope is operated on the island of La Palma by the Royal Greenwich Observatory in the Spanish Observatorio del Roque de los Muchachos of the Instituto de Astrofísica de Canarias. We acknowledge Dr. J. Knapen for his help during the observations. This work was partially supported by the Spanish DGICYT (Dirección General de Investigación Científica y Técnica) via Grants PB91-0525, PB94-1107 and PB97-0219. This research has made use of the NASA/IPAC Extragalactic Database (NED) which is operated by the Jet Propulsion Laboratory, California Institute of Technology, under contract with the National Aeronautics and Space Administration. C. Heller acknowledges support from DFG grant Fr 325/39-1, 39-2.

\section{References}

Beckman J.E., Rozas M., Zurita A., Knapen J.H., 1999, ApJ (submitted)

Blackman C.P., 1983, MNRAS 202, 379

Cepa J., Beckman J.E., 1989, A\&AS 79, 4

Cepa J., Beckman J.E., 1990, A\&AS 83, 211

de Vaucouleurs G., de Vaucouleurs A., Corwin H.G., et al., 1991, Third Reference Catalogue of Bright Galaxies (RC3). Springer, New York

Devereux N.A., Young J.S., 1991, ApJ 371, 515

Ferguson A.M.N., Wyse R.F.G., Gallagher J.S., Hunter D.A., 1996, AJ 111, 2265

Filippenko A.V., Greenstein J.L., 1984, PASP 96, 530

Fuentes O., 1997, PhD thesis. Univ. La Laguna

Hodge P.W., 1976, ApJ 205, 728

Hodge P.W., 1987, PASP 99, 915

Kennicutt R.C., 1984, ApJ 287, 116

Kennicutt R.C., 1989, ApJ 344, 685

Kennicutt R.C., 1992, in Star formation in Stellar systems, Tenorio-Tagle G. et al. (eds.). Cambridge Univ. Press, Cambridge

Kennicutt R.C., Edgar B.K., Hodge P.W., 1989, ApJ 337, 761

Knapen J.H., Arnth-Jensen N., Cepa J., Beckman J.E., 1993, AJ 106, 56

Knapen J.H., 1998, MNRAS 297, 255

Laine S., 1997, BAAS 29, 1345

Laine S., Gottesman S.T., 1998, MNRAS 297, 1041

Laine S., Heller C.H. (in preparation)

Massey P., Lang G.C., DeGioia-Eastwood K., Garmany C.D., 1995, ApJ 438, 188

Mihos J.C., Hernquist L., 1994, ApJ 425, L13

Oke J.B., 1974, ApJS 27, 21

Osterbrock D.E., 1974, Astrophysics of gaseous nebulae. Freeman, San Francisco

Prieto M., Aguerri J.A.L., Varela A.M., Muñoz-Tunón C., 1998, AJ (submitted)

Rand R.J., 1992, AJ 103, 815

Rozas M., Beckman J.E., Knapen J.H., 1996a, A\&A 307, 735

Rozas M., Knapen J.H., Beckman J.E., 1996b, A\&A 312, 275

Rozas M., Castañeda H.O., Beckman J.E., 1998, A\&A 330, 873

Spitzer L., 1978, Physical Processes in the Interstellar Medium. Wiley, New York

Stone R.P.S., 1977, ApJ 218, 767

Vacca W.D., Garmany C.D., Shull J.M., 1996, ApJ 460, 914

van den Bergh S., 1981, AJ 86, 1464

Walterbos R.A.M., Braun R., 1992, A\&A 92, 625

Ye T., 1992, MNRAS 255, 32

Zaritzky D., Kennicutt R.C., Huchra J.P., 1994, ApJ 420, 87

Zurita A., Rozas M., Beckman J.E., 1999 (in preparation) 